\title{
Perfil de enfermeiros-professores da educação profissional técnica de nivel médio em enfermagem
}

\author{
Profile of nurses-teachers of medium-level technical professional education in nursing \\ Perfil de enfermeros-profesores de la educación profesional técnica de nivel medio en enfermería
}

\begin{abstract}
RESUMO
Objetivo: Descrever o perfil dos enfermeiros-professores atuantes na educação profissional de nível médio em Enfermagem no estado do Rio Grande do Sul. Método: Após aprovação ética, os dados foram coletados através de questionário online, respondido por 109 docentes. Análise realizada à luz da estatística quantitativa e descritiva, mediante frequência, porcentagem, e análise de frequências cruzadas. Resultados: Predominância do sexo feminino, maiores de 31 anos, graduados de 5 a 10 anos, pós-graduados, não licenciados, com experiência docente menor que 5 anos e assistencial entre 5 a 10 anos (51,4\%), ou superior a 10 anos $(25,7 \%$ ). Os enfermeiros-professores trabalham predominantemente sob vínculo da Consolidação das Leis do TrabaIho (CLT), possuem duplo vínculo empregatício e, em sua maioria, consideram a docência como atividade profissional complementar. Conclusões: Os enfermeiro-professores são profissionais experientes na prática do que ensinam e são dispostos para seu aprimoramento profissional, porém ainda apresentam pouca formação e experiência docente.

DESCRITORES: Docentes de Enfermagem, Educação em Enfermagem, Educação Técnica em Enfermagem, Enfermagem, Docentes.
\end{abstract}

\section{ABSTRACT}

Objective: To describe the profile of nurses-teachers working in professional nursing education in the State of Rio Grande do Sul. Method: After ethical approval, data were collected through an online questionnaire, answered by 109 teachers. Analysis performed in the light of quantitative and descriptive statistics, using frequency, percentage, and cross-frequencies analysis. Results: Predominance of the female gender, over 31 years of age, graduates from 5 to 10 years old, post-graduates, unlicensed, less than 5 years of teaching experience and 5 to 10 years of care $(51.4 \%)$, or higher than 10 years $(25.7 \%)$. The nurses-teachers work predominantly under the Consolidation of Labor Laws (CLL), have a double employment relationship and, for the most part, consider teaching as a complementary professional activity. Conclusions: The nurses-teachers are professionals who are experienced in the practice of what they teach and are prepared for their professional improvement, but still have little training and teaching experience.

DESCRIPTORS: Faculty Nursing, Education Nursing, Education Nursing Associate, Nursing, Teaching.

\section{RESUMEN}

Objetivo: Describir el perfil de los enfermeros-profesores actuantes en la educación profesional de nivel medio en Enfermería en el estado de Rio Grande do Sul. Método: Después de la aprobación ética,los datos se recopilaron a través de un cuestionario online, respondido por 109 docentes. Análisis realizado a la luz de la estadística descriptiva, a menudo, porcentaje, y análisis de frecuencias cruzadas. Resultados: Predominancia del sexo femenino, mayores de 31 años, graduados de 5 a 10 años, postgraduados, no licenciados, con experiencia docente menor que 5 años y asistencial entre 5 a 10 años (51,4\%), o superior a 10 años $(25,7 \%$ ). Los enfermeros-profesores trabajan predominantemente bajo vínculo de la Consolidación de las Leyes del Trabajo (CLT), poseen doble vínculo laboral y, en su mayoría, consideran la docencia como actividad profesional complementaria. Conclusiones: Los enfermeros-profesores son profesionales experimentados en la práctica de lo que enseñan y están dispuestos para su perfeccionamiento profesional, pero todavía presentan poca formación y experiencia docente.

DESCRIPTORES: Docentes de Enfermería, Educación en Enfermería, Graduación en Auxiliar de Enfermería, Enfermería, Docentes.

RECEBIDO EM: 16/08/2020 APROVADO EM: 25/08/2020

\section{Miriam Trombetta Franco}

Mestre em Ensino na Saúde pela Universidade Federal de Ciências da Saúde de Porto Alegre (UFCSPA), Especialista em Terapia Intensiva, Emergência e Trauma pelo Instituto de Educação e Pesquisa do Hospital Moinhos de Vento e Especialista em Geriatria e Gerontologia pela Faculdade Unyleya. Coordenadora do Conselho Consultivo das Escolas Técnicas de Enfermagem do Rio Grande do Sul vinculado à Associação Brasileira de Enfermagem - seção Rio Grande do Sul (CCETE/ABEn-RS) e Enfermeira em Cuidados Domiciliares Avançados.

ORCID: 0000-0002-2191-187X 


\section{Morgana Thaís Carollo Fernandes}

Enfermeira. Pós-Doutoranda em Saúde da Criança pela Pontifícia Universidade Católica do Rio Grande do Sul (PUCRS), bolsista pela University of Toronto (UofT). Professora Colaboradora da Residência Multiprofissional em Saúde da Criança e Pesquisadora Associada do Programa de Extensão e Pesquisa em Saúde Urbana, Ambiente e Desigualdades da Universidade Federal do Rio Grande do Sul (UFRGS).

ORCID: 0000-0002-7989-294X

\section{Luzia Fernandes Millão}

Doutorado em Psicologia pela Universidade Pontifícia de Salamanca. Professor adjunto IV da Fundação Universidade Federal de Ciências da Saúde de Porto Alegre (UFCSPA). Presidente da Comissão de Residência Multiprofissional Integrada em Saúde da UFCSPA-ISCMPA. Professora do Mestrado Profissional Ensino na Saúde da UFCSPA Colaboradora do Instituto Nacional de Estudos e Pesquisas Educacionais Anísio Texeira.

ORCID: 0000-0003-3399-7428

\section{INTRODUÇÃO}

A formação do técnico de Enfermagem cada vez mais exige o desenvolvimento de competências para que ele seja capaz de articular, mobilizar e colocar em ação valores, conhecimentos e habilidades imprescindíveis para o desempenho eficiente e eficaz de atividades requeridas pela natureza do trabalho ${ }^{(1,2)}$. Inúmeras necessidades e desafios a serem enfrentados com relação à formação desses profissionais, inclusive em virtude das reconhecidas especificidades e peculiaridades que caracterizam o trabalho em saúde, em que os riscos associados envolvem agravos que podem perturbar a qualidade de vida da população e dos próprios trabalhadores ${ }^{(3)}$.

Estudos indicam que a educação profissional técnica de Enfermagem exibe falhas, déficits e dificuldades frente às amplas questões relacionadas aos diversos aspectos envolvidos no processo de formação desses profissionais ${ }^{(3-9)}$. Uma formação deficitária, que libera no mundo de trabalho pessoas sem o devido preparo, acaba por gerar baixa qualidade da assistência à saúde e também alto custo, uma vez que é preciso gerenciar as falhas advindas dos processos por eles operados e, ainda, buscar e, talvez, preparar outro profissional ${ }^{(8)}$.

Intrinsecamente envolvido nessa problemática, encontra-se o enfermeiro-professor, uma vez que é o responsável direto pelo processo de formação dos profissionais de nível médio em Enfermagem. Mas, ao longo da história, pouco foi investido na formação dos enfermeiros-professores para essa área no Brasil ${ }^{(10)}$. Atividades docentes constituem-se em pontos frágeis no processo de profissionalização, uma vez que, a formação na graduação em Enfermagem não é voltada para o exercício da docência, mas sim para o cuidado assistencial, e ainda em posição invertida, em que o graduando aprende a ser enfermeiro e não técnico de Enfermagem ${ }^{(3,5,7)}$.

O enfermeiro-professor é responsável por desenvolver uma proposta pedagógica que contemple a atuação voltada para a autonomia do educando, mediante modelos de ensino que reflitam o dia a dia do profissional com base em projeto político-pedagógico elaborado, executado e avaliado com a efetiva participação de todos os agentes educacionais, em especial os docentes ${ }^{(5,11)}$. Para formar profissionais éticos, comprometidos e imbuídos de espírito científico, o professor deve também ser dotado dessas mesmas qualidades. Os conteúdos necessitam ser integrados na realidade vivida, e os métodos devem estabelecer coerência entre a teoria e a prática, revelando uma abordagem pedagógica condizente com a práxis reflexiva, propiciando a aprendizagem significativa e conduzindo à educação transformadora ${ }^{(6,12)}$. O objetivo desse estudo foi descrever o perfil dos enfermeiros-professores da educação profissional técnica de nível médio em Enfermagem, em uma população de profissionais atuantes no estado do Rio Grande do Sul.

\section{METODOLOGIA}

Trata-se de um estudo quantitativo, com dados coletados entre novembro de 2016 a setembro de 2017, através de questionário online, respondido por enfermeiros-professores atuantes na educação profissional de nível médio em Enfermagem no estado do Rio Grande do Sul. Para a captação de participantes foi utilizada a técnica em cadeia snowball. O questionário foi enviado para 197 endereços eletrônicos, sendo respondido por 109 enfermeiros-professores atuantes na educação profissional de nível médio em Enfermagem no Estado do Rio Grande do Sul. Como critério de inclusão, os participantes deveriam ter experiência com supervisão de turmas em estágio técnico de Enfermagem obrigatório nos serviços de saúde nos últimos cinco anos. Todas as exigências éticas de pesquisas desenvolvidas com seres humanos foram respeitadas neste estudo com base nas orientações e disposições da Resolução CNS/MS 466/2012 $2^{(15)}$.

A pesquisa foi iniciada após aprovação pelo Comitê de Ética e Pesquisa da Universidade Federal de Ciências da Saúde de Porto Alegre, sob o Certificado de Apresentação para Apreciação Ética (CAAE) n 61395816.6.0000.5345.

\section{RESULTADOS}

As mulheres foram preponderantes entre os docentes, compondo 87 (79,8\%) do total pesquisado. Quanto à faixa etária, evidenciou-se que 94 (86,3\%) eram 


\section{Tabela 1 - Distribuição dos enfermeiros docente segundo faixa etária.}

$$
\text { Variável }
$$

N

$\%$

Idade

21 a 25 anos

01

0,90

26 a 30 anos

14

12,8

31 a 40 anos

59

54,1

41 a 50 anos

22

20,2

51 a 60 anos

09

8,30

61 ou mais

04

3,70

Fonte: Dados da pesquisa, 2017

Figura 1 - Porcentagem de respondentes de acordo com a área de pós graduação e o tempo de experiência docente. Fonte: Dados da pesquisa, 2017.

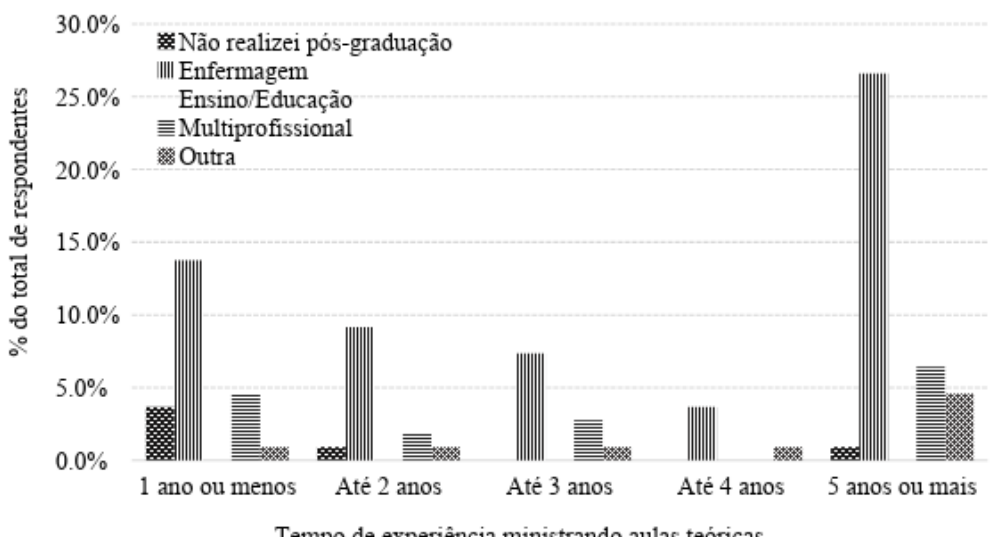

Figura 2 - Distribuição de respondentes de acordo formação pedagógica e tempo de conclusão da graduação em enfermagem.

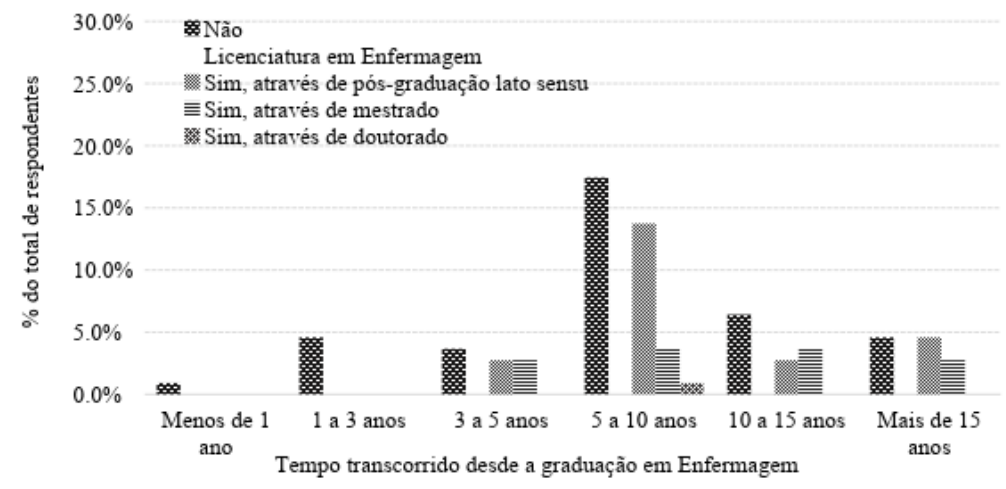

Fonte: Dados da pesquisa, 2017 maiores de 31 anos, e mais da metade dos participantes, $59(54,1 \%)$ correspondem à faixa etária compreendida entre 31 a 40 anos, sendo a distribuição variada entre outras idades, conforme Tabela 1.

Com relação ao tempo de graduação, 47 (43,1\%) dos enfermeiros-professores indicaram ter concluído sua graduação de 5 a 10 anos e 28 (25,7\%), entre 10 e 15 anos. Quase a totalidade dos pesquisados $103(94,5 \%)$ realizou pós-graduação, sendo que apenas $11(10,1 \%)$ na área de ensino ou educação. Verifica-se, ainda, que não há pós-graduados em ensino/educação entre os profissionais com experiência docente menor de 4 anos conforme a figura 1 .

Indagados sobre a formação pedagógica específica para a atuação docente, $27(24,8 \%)$ dos pesquisados declararam possuir licenciatura em Enfermagem; 15 $(13,7 \%)$ indicaram ter mestrado ou doutorado; $26(23,9 \%)$ pós-graduação Lato Sensu e ainda 41 (37,6\%) declararam não ter nenhum tipo de formação pedagógica. Do total, $27(24,8 \%)$ dos docentes possuem licenciatura. Relacionando-se a formação pedagógica com o tempo de graduação, evidenciou-se que a presença de docentes com licenciatura concentra-se entre os formados de 10 a 15 anos (50\%) conforme figura 2 .

Quando indagados sobre o tempo de atuação como docente, ministrando aulas teóricas, 49 (45\%) indicaram ter 5 anos ou mais de experiência, 25 (22,9\%) relataram ter 1 ano ou menos de experiência, 14 $(12,8 \%)$ até 2 anos, 12 (11\%) até 3 anos e $9(8,3 \%)$ até 4 anos. Com relação ao tempo de atuação como supervisor de estágio, 48 (44,1\%) têm 4 anos ou mais de experiência. Quanto a experiência docente especificamente, conclui-se que 60 (55\%) são novatos, apresentando menos de 5 anos na docência ministrando aulas teóricas; e 71 (65,1\%) são novatos na função docente de supervisão de estágio, com menos de 4 anos de atuação. Quanto ao tempo de experiência na Enfermagem assistencial, 56 $(51,4 \%)$ relatam possuir de 5 a 10 anos, enquanto $28(25,7 \%)$ possuem mais de 10 anos de experiência assistencial.

Em relação às condições de trabalho na 
instituição de ensino, a maioria dos pesquisados $65(59,6 \%)$ refere atuar mediante vínculo CLT e 44 (40,4\%) trabalham em Regime de Pagamento Autônomo (RPA) ou outro tipo de vínculo. No total, $68(62,4 \%)$ dos enfermeiros-professores pesquisados declararam que a profissão docente representa atividade profissional complementar. Entre os 65 enfermeiros-professores que atuam com vínculo CLT, $30(46,1 \%)$ compreendem sua prática docente como atividade profissional complementar, enquanto, entre os 33 enfermeiros-professores que atuam em regime de RPA, 28 (86,3\%) pensa da mesma forma. A maioria dos enfermeiros-professores 84 (77,1\%) possui vínculo trabalhista adicional, sendo que, destes, 41 (49,5\%) apresentam vínculo concomitante com uma instituição hospitalar. Relacionando-se o tipo de vínculo trabalhista dos docentes e o trabalho sob vínculo adicional, dentre os 65 trabalhadores em regime CLT, 43 (66\%) possuem algum vínculo de trabalho adicional e dentre os 33 trabalhadores via RPA, 31 (94\%) possuem vínculo adicional.

\section{DISCUSSÃO}

O perfil docente constituído predominantemente por mulheres, está de acordo com os dados apresentados na pesquisa nacional Perfil da Enfermagem que mostra que $84,6 \%$ dos enfermeiros são do sexo feminino; e com os achados de outros estudos que investigaram as características de enfermeiros-professores da educação profissional ${ }^{(10,16-21)}$. Os dados relacionados ao tempo de graduação no curso de Enfermagem são similares aos encontrados em outros estudos sobre o tema ${ }^{(10,18-19,22)}$. O fato de quase a totalidade dos pesquisados $103(94,5 \%)$ ter realizado pós-graduação denota uma preocupação dos docentes com seu próprio aprimoramento profissional. Entretanto, o fato de somente os profissionais com experiência docente acima de 4 anos procurarem a formação na área de ensino/educação possivelmente indica que este tema de pós-graduação só começa a ser pensado após um caminho
Entretanto, o

fato de somente

os profissionais

com experiência

docente acima de

4 anos procurarem

a formação na área

de ensino/educação

possivelmente

indica que este tema

de pós-graduação

só começa a ser

pensado após

um caminho já

percorrido pelo

profissional dentro

da docência. já percorrido pelo profissional dentro da docência ${ }^{(10)}$.

Percebe-se uma situação intrincada com relação ao baixo percentual de licenciados e a atuação de profissionais que declaram não ter nenhum tipo de formação pedagógica, pois a resolução $\mathrm{CNE} / \mathrm{CEB}$ 6/2012 $2^{(23)}$, que define Diretrizes Curriculares Nacionais para a Educação Profissional Técnica de Nível Médio, traz, em seu artigo 40, que a formação inicial para a docência nesse nível de educação realiza-se em cursos de graduação e programas de licenciatura ou sob outras formas, de acordo com a legislação e as normas estabelecidas pelo Conselho Nacional de Educaçãoo ${ }^{(13,23)}$.

Segundo Gomes ${ }^{(13)}$, tal resolução permite um espaço demasiado, ao conceder prazo até 2020 para que professores graduados, não licenciados, em efetivo exercício docente, tenham reconhecidos seus saberes profissionais mediante pós-graduação Lato Sensu, de caráter pedagógico, podendo se considerar equivalente às licenciaturas ${ }^{(23)}$. Atualmente muitos profissionais permanecem atuando na formação profissional sem preparo pedagógico, após o prazo supracitado, cada enfermeiro atuando nesse nível de formação ver-se-á compelido a uma avaliação pessoal sobre o investimento em seu fazer profissional, quando será preciso considerar múltiplas questões relacionadas aos rumos da carreira docente, dentre elas a valorização profissional, as perspectivas de progressão, as condições de trabalho e o custo-benefício do investimento.

Sobre a formação pedagógica através de licenciatura, segundo as Diretrizes Curriculares Nacionais para os Cursos de Graduação em Enfermagem (DCN/2001), a estrutura do curso deve assegurar a articulação da graduação com a licenciatura em Enfermagem. As diretrizes indicam que mesmo havendo a possibilidade da licenciatura na área, o curso de graduação em Enfermagem deve seguir a orientação generalista, contemplando, em seu conteúdo essencial, o "Ensino de Enfermagem", que garantiria capacitação pedagógica ao enfermeiro, independentemente da licenciatura ${ }^{(24)}$.

Mas, apesar de as DCN/2001 detalha- 
rem os princípios, fundamentos e condições da formação no modelo de bacharel em Enfermagem, incluindo temáticas relacionadas à atividade de ensino inerente à profissão, sabe-se que, na prática, não existe, nos cursos de graduação, uma preocupação oficial quanto à formação para a docência ${ }^{(5,24-25)}$. Ainda os cursos de licenciatura em Enfermagem enfrentam queda na procura, já não sendo mais possível encontrar com facilidade essa modalidade de formação disponível em modo presencial, restando apenas a opção de formação pedagógica em curso de pós-graduação ${ }^{(14)}$.

Relativo ao número de licenciados, nos estudos de Frozoni e Silva ${ }^{(10)}$, Silva ${ }^{(17)}$, Frozoni e Silva ${ }^{(20)}$ e Coloni ${ }^{(21)}$, o percentual de enfermeiros-professores com licenciatura varia de $21,9 \%$ a $34,8 \%$, sendo esses dados semelhantes aos aqui verificados. Backes et al. ${ }^{(22)}$ de forma contrária constatou que $56,1 \%$ dos docentes eram licenciados, contudo analisou predominantemente professores de escolas públicas e, segundo os próprios autores, não reflete as mesmas características de formação e trabalho de outros tipos de instituições de ensino. A concentração de licenciados entre os formados de 10 a 15 anos atrás e a ausência de enfermeiros-professores formados há menos de 5 anos confirmam a escassa oferta desses cursos, relatada por outros estudos ${ }^{(14,21)}$.

Chama atenção nos achados a presença de enfermeiros com titulação Stricto Sensu e o fato de 15 (13,7\%) dos participantes indicarem possuir formação pedagógica, através da realização de mestrado ou doutorado, isto porque é sabido que os cursos de pós-graduação Stricto Sensu muitas vezes se voltam para a formação de pesquisadores ${ }^{(21)}$. Em outros estudos, o percentual de mestres varia de $7 \%$ ( 1 participante) na pesquisa de Dias et al.18, a $41,4 \%$ (17 participantes) na pesquisa de Backes et al. ${ }^{(22)}$, sendo também recorrente a presença de doutores nos espaços de formação profissional pesquisados ${ }^{(10,18,20-21)}$. Quanto ao tempo de experiência assistencial e o tempo transcorrido desde a graduação, o estudo de Backes et al. ${ }^{(22)}$, indica a crença de que a maioria dos docentes enfermeiros é recém-formada e com pouca experiência assistencial em Enfermagem; contudo, esse pensamento não condiz com os achados na presente pesquisa e em outras sobre o tema ${ }^{(10,13,18,22)}$.

A presença da maioria de profissionais atuando mediante vínculo de Consolidação das Leis de Trabalho (CLT), considerado mais estável, é um fator muito positivo que abre possibilidade de estímulo ao investimento do docente em sua formação

\section{Chama atenção nos}

achados a presença

de enfermeiros

com titulação

Stricto Sensu e o

fato de $15(13,7 \%)$

dos participantes

indicarem

possuir formação

pedagógica

e atuação profissional. A associação entre o vínculo de trabalho e a valorização profissional evidencia-se na medida em que uma ínfima porcentagem de docentes (5,5\%) trabalha em regime RPA/outro e, simultaneamente, considera a docência como sua atividade profissional principal.

Percebe-se que a relação formalizada como CLT faz com que a maioria dos profissionais encare seu fazer docente como atividade profissional principal, mas é notório nos resultados que, mesmo nesse grupo (CLT), ainda muitos consideram a atividade docente como complementar. Então se pode inferir que prevalece entre os pesquisados a visão da prática docente como algo pouco estabelecido, informal ou menos importante, visto que a maioria encara a profissão docente como atividade profissional complementar. A prática de atuação profissional mediante mais de um vínculo empregatício aparece como característica de enfermeiros-professores também em outros estudos, com uma variação de $64 \%$ a $89,4 \%$. Tal situação é considerada preocupante, tendo em vista que a prática pedagógica é uma atividade complexa, demandando tempo de preparo cotidiano que extrapola a carga horária presencial do professor ${ }^{(10,13,19,22)}$.

E novamente o vínculo trabalhista menos formalizado ganha destaque negativo, ao se relacionar com a característica de vínculo adicional, pois, diferentemente dos professores sob CLT, quase todos os docentes que trabalham em regime RPA/outro possuem vínculo de trabalho adicional, corroborando com as análises de Backes et al. ${ }^{(22)} \mathrm{e}$ Sgarbi et al. ${ }^{(26)}$ ao levantarem a hipótese do duplo vínculo empregatício estar relacionado à precarização do ensino profissional, inclusive mediante ao vínculo não estável.

Mediante o exposto, percebe-se o enfermeiro-professor da educação profissional técnica de nível médio em Enfermagem com adequada experiência assistencial disposto para seu aprimoramento profissional, porém ainda com pouca formação pedagógica e experiência docente, carecendo de estabilidade, perspectiva e valorização profissional. Esta pesquisa apresenta algumas limitações, principalmente relacionadas à dificuldade de contatar as escolas profissionais e seus docentes, tendo sido então utilizada a técnica snowball, que apesar de ampliar o número de participantes, pode não garantir representatividade de toda população. Outra limitação se encontra no fato de não haver a disponibilidade de informações atualizadas, relativas ao número de enfermeiros-professores atuantes no estado, o que acarretou na ausência de cálculo amostral para a coleta de dados. 


\section{artigo}

\section{CONCLUSÕES}

Com este estudo, espera-se trazer à tona elementos que merecem reflexão e discussão e que também possam fomentar estratégias promotoras da valoriza- ção e qualificação da atuação docente de Enfermagem nos cursos de formação profissional técnica. Salienta-se que revelar o perfil dos enfermeiros-professores pode permitir contribuições não só para a Enfermagem como profissão, mas também para toda sociedade que de maneira direta é afetada pelos benefícios ou malefícios advindos da formação do maior grupo profissional atuante no setor saúde do país.

\section{REFERÊNCIAS}

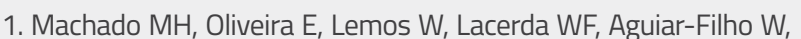
Wermelinger $\mathrm{M}$, et al. Mercado de trabalho da enfermagem: aspectos gerais. Enferm Foco. 2016 mar; 7(n.esp.): 35-62.

2. Brasil. CNE/CEB. Parecer $n^{\circ}$ 16, de 05 de outubro de 1999. Diretrizes Curriculares Nacionais para a Educação Profissional de Nível Técnico. Brasília, DF: Ministério da Educação; 1999.

3. Lessmann JC, Lanzoni GMM, Gubert E, Mendes PXG, Prado ML, Backes VM. Educação profissional em enfermagem: necessidades, desafios e rumos. Rev Min Enferm. 2012; 16(1): 106-10.

4. Camargo RAA, Gonçalves AE, Góes FSN, Nakata CY, Pereira MCA. Assessment of the training of nursing technicians by nurses who work in hospitals . Rev Min Enferm. 2015; 19(4): 951-964.

5. Ferreira-Júnior MA. Os reflexos da formação inicial na atuação dos professores enfermeiros. Rev Bras Enferm. 2008; 61: 866-71.

6. Góes FSN, Côrrea AK, Camargo RAA, Hara CYN. Learning needs of Nursing students in technical vocational education. Rev Bras Enferm. 2015; 68: 20-5.

7. Guberti E, Prado MLD. Desafios na prática pedagógica na educação profissional em enfermagem. Rev Eletr Enferm [Internet]. 2011 [acesso em 2017 set 30]; 13: 285-95. Disponivel em: https://www. fen.ufg.br/revista/v13/n2/pdf/v13n2a15.pdf

8. Silva ACAB, Silva GTR, Silva RMO, Vieira SL, Santana MS. Secondary professional education: characterization of scientific production in graduate studies. Acta Paul Enferm. 2013; 26: 499-505.

9. Vieira SL, Silva GTR, Fernandes JD, Silva ACAB, Santana MS, Santos TBS. Des-interesse no ensino profissionalizante na produção do Seminário Nacional de Diretrizes para a Educação em Enfermagem. Rev Bras Enferm. 2014; 67(1): 141-8.

10. Frozoni RC, Souza MCBDME. Educação profissional técnica de nível médio em enfermagem: perfil socioeconômico dos professores de um município do estado de São Paulo. Rev Eletr Gestão Saúde [Internet]. 2013 [acesso em 2017 set 30]; 1(1). Disponível em: http://periodicos.unb. br/index.php/rgs/article/view/22 968/0.

11. Brasil. Ministério da Educação. Educação profissional: referenciais curriculares nacionais da educação profissional de nível técnico: área Saúde. Brasília, DF: Ministério da Educação; 2000.

12. Feuerwerker LCM, Cecilio LCO. O hospital e a formação em saúde: desafios atuais. Ciênc Saúde Coletiva. 2007; 12: 965-71.

13. Gomes VCO. Perfil dos docentes da educação profissional técnica de nível médio em enfermagem. Dissertação (mestrado) Universidade Federal da Bahia, Escola de Enfermagem da Universidade Federal da Bahia; 2016.

14. Spessoto MMRL, Silva MLM, Real GCM, Missio L. Docência: a vi- vência de bacharéis enfermeiros. Horizontes: Rev Educ. 2017; 3(6): 60-77.

15. Brasil. Resolução $n^{\circ}$ 466, de 12 de dezembro de 2012. Aprova as diretrizes e normas regulamentadoras de pesquisas envolvendo seres humanos. Brasilia, DF: CNS; 2012.

16. Fundação Oswaldo Cruz. Pesquisa inédita traça perfil da enfermagem no Brasil [Internet]. Rio de Janeiro: Fiocruz; 2015 [acesso em: 2016 nov 26]. Disponível em: http://portal.fiocruz.br

17. Silva MFD, Braun JR, Lima ITCD. Caracterização do perfil dos professores que atuam no curso técnico de enfermagem no colégio estadual Jorge Schimmelpfeng da cidade de Foz do Iguaçu. Rev Eletr Cien Inov Tecnol [Internet]. 2015 [acesso em 201710 set]; 1(1). Disponivel em: https://periodicos.utfpr.edu.br/recit/article/ view/4269/pdf

18. Souza EG, Presoto LH. O perfil dos docentes do ensino técnico profissionalizante em enfermagem. Rev Cient Enferm. 2013; 3(9): 23-30.

19. Dias EP, Stutz BL, Resende TC, Batista NB, Sene SS. Expectativas de alunos de enfermagem frente ao primeiro estágio em instituições de saúde. Rev Psicoped. 2014; 31(94): 44-55.

20. Frozoni RC, Souza MCBDME. Identidade profissional e perfil dos professores dos cursos de educação profissional técnica de nível médio em enfermagem de um município do interior do Estado de São Paulo. Dissertação (Mestrado). São Paulo: Escola de Enfermagem de Ribeirão Preto; 2013.

21. Coloni CSM, Teixeira VM, Moreira MCO, Piotto R, Góes FSN, Camargo RAA. Pedagogical practice in mid-level professional nursing education. Cogit Enferm. 2016 jan-mar; 21(1): 1-9.

22. Backes VMS, Menegaz JC, Francisco BS, Reibnitz KS, Costa LM. Características de formação e trabalho de professores de nível médio em enfermagem. Rev Rene. 2014 nov-dez; 15(6): 957-63.

23. Brasil. Resolução CNE/CEB n 6, de 20 de setembro de 2012. Define Diretrizes Curriculares Nacionais para a Educação Profissional Técnica de Nível Médio. Brasilia, DF: CNE/CEB; 2012.

24. Brasil. Resolução CNE/CES n 3, de 07 de novembro de 2001. Diretrizes Curriculares Nacionais do Curso de Graduação em Enfermagem. Brasilia, DF: Ministério da Educação; 2001.

25. Duarte CG, Lunardi VL, Barlem ELD. Satisfaction and Suffering in the work of the nursing teacher: an integrative review. Rev Min Enferm. 2015; 20:e939.

26. SGARBI, Aniandra Karol Gonçalves et al. Enfermeiro docente no ensino técnico em enfermagem. Laplage em Revista. 2018, v. 4, n. 1, p. 254-273. 